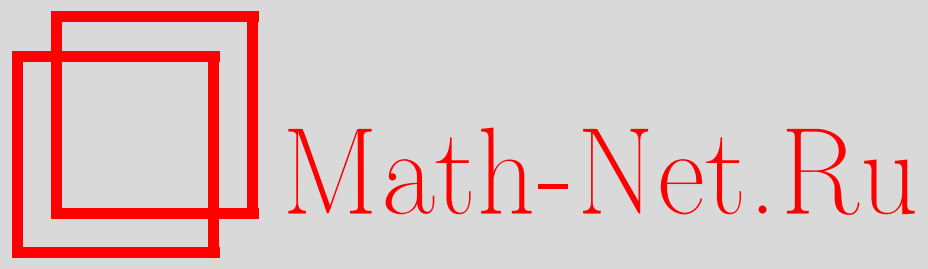

О. А. Кривошеева, А. С. Кривошеев, Критерий выполнения фундаментального принципа для инвариантных подпространств в ограниченных выпуклых областях комплексной плоскости, Функи. анализ и его прил., 2012, том 46, выпуск 4, 14-30

DOI: https://doi.org/10.4213/faa3086

Использование Общероссийского математического портала Math$\mathrm{Net.Ru}$ подразумевает, что вы прочитали и согласны с пользовательским соглашением

http://www . mathnet.ru/rus/agreement

Параметры загрузки:

IP : 54.224 .60 .19

26 апреля 2023 г., 15:43:31

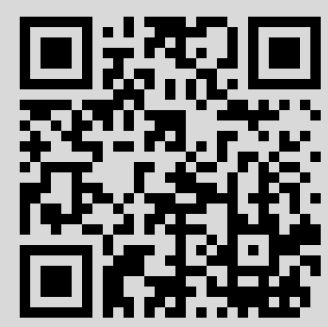




\title{
Критерий справедливости фундаментального принципа для инвариантных подпространств в ограниченных выпуклых областях комплексной плоскости*
}

\author{
(c) 2012. О. А. КривошеевА, А. С. Кривошеев
}

\begin{abstract}
В работе изучается проблема справедливости фундаментального принципа для инвариантных относительно оператора дифференцирования подпространств функций, аналитических в ограниченной выпуклой области комплексной плоскости, допускающих спектральный синтез. Ранее эта проблема была решена при одном ограничении на кратность собственных значений оператора дифференцирования. В данной работе это ограничение снимается. Таким образом, приводится полное решение проблемы фундаментального принципа для произвольных нетривиальных замкнутых инвариантных подпространств, допускающих спектральный синтез, в произвольных ограниченных выпуклых областях.
\end{abstract}

\section{$\S 1$. Введение}

Пусть $D$ - выпуклая область в комплексной плоскости $\mathbb{C}, H(D)$ - пространство функций, аналитических в области $D$, с топологией равномерной сходимости на компактных подмножествах из $D, H^{*}(D)$ - пространство, сильно сопряженное к $H(D)$, называемое также пространством аналитических функционалов. Символом $W$ будем обозначать нетривиальное замкнутое подпространство в $H(D)$, инвариантное относительно оператора дифференцирования, т. е. вместе с каждой функцией $\varphi$ подпространство $W$ содержит и ее производную $\varphi^{\prime}$. Поскольку $W$ нетривиально, по теореме Хана-Банаха найдется ненулевой аналитический функционал $\mu \in H^{*}(D)$, который обращается в нуль на всех функциях подпространства $W$. Пусть

$$
f(\lambda)=(\mu, \exp (\lambda z)), \quad \lambda \in \mathbb{C},
$$

- преобразование Лапласа функционала $\mu$. Известно (см., например, $[1, \S 12$, п. 1]), что $f(\lambda)$ - целая функция экспоненциального типа (другими словами, выполняется неравенство

$$
|f(\lambda)| \leqslant A \exp (B|\lambda|), \quad \lambda \in \mathbb{C},
$$

где $A$ и $B$ - некоторые положительные постоянные). Множество всех экспонент полно в пространстве $H(D)$ (см., например, [1, §12, п. 1]). Поэтому функция $f(\lambda)$ отлична от тождественного нуля.

Если $\xi$ - собственное значение оператора дифференцирования в $W$ (т.е. собственная функция $\exp (\xi z)$ этого оператора лежит в $W)$, то

$$
f(\xi)=(\mu, \exp (\xi z))=0 .
$$

Следовательно, совокупность всех собственных значений $\left\{\lambda_{k}\right\}$ оператора дифференцирования в $W$ является частью нулевого множества целой функции $f$.

\footnotetext{
*Работа поддержана РФФИ (грант 10-01-00233-а).
} 
Это, в частности, означает, что множество $\left\{\lambda_{k}\right\}$ конечно или счетно. В последнем случае $\left|\lambda_{k}\right| \rightarrow \infty$ при $k \rightarrow \infty$. Будем считать, что последовательность $\left\{\lambda_{k}\right\}$ пронумерована по неубыванию модулей.

Пусть $m_{k}$ - кратность собственного значения $\lambda_{k}$ в $W$, т. е. присоединенные функции $z^{n} \exp \left(\lambda_{k} z\right)$ оператора дифференцирования принадлежат подпространству $W$ для всех $n=1, \ldots, m_{k}-1$, а функция $z^{m_{k}} \exp \left(\lambda_{k} z\right)$ таким свойством уже не обладает. Тогда

$$
f^{(n)}\left(\lambda_{k}\right)=\left.(\mu, \exp (\lambda z))\right|_{\lambda=\lambda_{k}} ^{(n)}=\left(\mu, z^{n} \exp \left(\lambda_{k} z\right)\right)=0, \quad n=0, \ldots, m_{k}-1 .
$$

Следовательно, число $m_{k}$ не превосходит кратности нуля $\lambda_{k}$ функции $f(\lambda)$. В этом случае говорят, что кратная последовательность $\Lambda=\left\{\lambda_{k}, m_{k}\right\}_{k=1}^{\infty}$ (которую называют кратным спектром оператора дифференцирования в подпространстве $W$ ) является частью кратного нулевого множества функции $f$. Пусть

$$
E(\Lambda)=\left\{z^{n} \exp \left(\lambda_{k} z\right)\right\}_{k=1, n=0}^{\infty, m_{k}-1}
$$

- множество всех собственных и присоединенных функций оператора дифференцирования в $W$. Говорят, что подпространство $W$ допускает спектральный синтез, если система $E(\Lambda)$ полна в нем. Примерами таких инвариантных подпространств служат пространства решений однородных уравнений свертки [3]

$$
M(\varphi)(\varsigma) \equiv(\nu, \varphi(z+\varsigma)) \equiv \int \varphi(z+\varsigma) d \sigma(z) \equiv 0,
$$

где $\nu$ - некоторый функционал из $H^{*}(D)$, а $d \sigma-$ соответствующая ему (не единственным образом) комплексная мера с компактным носителем в области $D$. В частности, это относится к линейным дифференциальным уравнениям с постоянными коэффициентами как конечного, так и бесконечного порядков, к линейным разностным и дифференциально-разностным уравнениям с постоянными коэффициентами как конечного, так и бесконечного порядков (см., например, $[1, \S 17$, п. 1]). В случае когда подпространство $W$ является пространством решений однородного линейного дифференциального уравнения с постоянными коэффициентами, хорошо известен фундаментальный принцип Л. Эйлера, согласно которому каждая функция из $W$ представляет собой линейную комбинацию собственных и присоединенных функций оператора дифференцирования в $W$ (их в этом случае конечное число). В связи с этим проблема представления функций из инвариантного подпространства $W \subset H(D)$, допускающего спектральный синтез, посредством рядов

$$
\sum_{k=1, n=0}^{\infty, m_{k}-1} d_{k, n} z^{n} \exp \left(\lambda_{k} z\right)
$$

по собственным и присоединенным функциям оператора дифференцирования в $W$, сходящихся в топологии пространства $H(D)$, носит также название проблемы фундаментального принципа для инвариантных подпространств.

Ее решение тесно связано с решением интерполяционной задачи в пространствах целых функций и имеет очень богатую историю. Обзор некоторых основных результатов по проблемам фундаментального принципа и интерполяции можно найти в работе [4]. Здесь мы отметим лишь некоторые из работ, а именно 
[4]-[14], в которых решалась проблема фундаментального принципа. В работе [4] при условии $m(\Lambda)=0$, где

$$
m(\Lambda)=\varlimsup_{k \rightarrow \infty} \frac{m_{k}}{\left|\lambda_{k}\right|},
$$

найдено полное решение проблемы фундаментального принципа для произвольных нетривиальных замкнутых инвариантных подпространств, допускающих спектральный синтез, в произвольных выпуклых областях комплексной плоскости. Целью данной работы является доказательство того, что в случае ограниченной области $D$ условие $m(\Lambda)=0$ необходимо для справедливости фундаментального принципа. Таким образом, результат этой работы вместе с результатом работы [4] дает полное решение проблемы фундаментального принципа для произвольных нетривиальных замкнутых инвариантных подпространств, допускающих спектральный синтез, в произвольных ограниченных выпуклых областях уже без всяких дополнительных ограничений.

\section{§2. Замкнутость множества сумм рядов экспоненциальных мономов}

Прежде чем перейти к формулировке и доказательству основного результата этого параграфа, сделаем некоторые необходимые замечания и докажем вспомогательный результат.

Для последовательности $\Lambda=\left\{\lambda_{k}, m_{k}\right\}_{k=1}^{\infty}$ положим

$$
\sigma(\Lambda)=\varlimsup_{j \rightarrow \infty} \frac{\ln j}{\left|\xi_{j}\right|}, \quad N(\Lambda)=\varlimsup_{j \rightarrow \infty} \frac{j}{\left|\xi_{j}\right|},
$$

где $\left\{\xi_{j}\right\}$ - неубывающая по модулю последовательность, составленная из точек $\lambda_{k}$, причем каждая из точек $\lambda_{k}$ встречается в ней ровно $m_{k}$ раз.

Пусть система

$$
E(\Lambda)=\left\{z^{n} \exp \left(\lambda_{k} z\right)\right\}_{k=1, n=0}^{\infty, m_{k}-1}
$$

неполна в пространстве $H(D)$ и $W$ - подпространство в $H(D)$, которое является замыканием в топологии пространства $H(D)$ линейной оболочки системы $E(\Lambda)$. Тогда оно, очевидно, нетривиально (т. е. $W \neq\{0\}, H(D)$ ), замкнуто в $H(D)$, инвариантно относительно оператора дифференцирования и допускает спектральный синтез. Таким образом можно получить любое нетривиальное замкнутое и инвариантное относительно оператора дифференцирования подпространство пространства $H(D)$, допускающее спектральный синтез. При этом система $E(\Lambda)$ совпадает с множеством всех собственных и присоединенных функций оператора дифференцирования в $W$. Во введении отмечалось, что последовательность $\Lambda$ является частью нулей целой функции экспоненциального типа $f$. Следовательно, нули функции $f$, а значит, и последовательность $\Lambda$ имеют конечную верхнюю плотность (см., например, [15, гл. 1, теорема 2.3]), т.е. $N(\Lambda)<\infty$. Отсюда легко следует, что величина $\sigma(\Lambda)$ равна нулю.

При помощи функции $f(\lambda)$ всегда можно построить (см., например, [4], [15]) последовательность целых функций $\left\{f_{k, n}\right\}$, элементы которой являются преобразованиями Лапласа соответствующих элементов последовательности функционалов $\left\{\mu_{k, n}\right\} \subset H^{*}(D)$, биортогональной к системе функций $E(\Lambda)$, т. е. вы- 
полнены равенства

$$
\left(\mu_{k, n}, z^{p} \exp \left(\lambda_{j} z\right)\right)= \begin{cases}1, & \text { если } k=j, n=p \\ 0 & \text { в противном случае. }\end{cases}
$$

Пусть $W(D, \Lambda)$ - пространство сумм рядов вида $(1)$, которые сходятся в топологии пространства $H(D)$. Существование биортогональной последовательности обеспечивает единственность представления элемента $g \in W(D, \Lambda)$ рядом (1), поскольку в этом случае коэффициенты ряда однозначно определяются по формуле

$$
d_{k, n}=\left(\mu_{k, n}, g\right), \quad k=1,2, \ldots, n=0,1, \ldots, m_{k}-1 .
$$

Из определений множеств $W(D, \Lambda)$ и $W$ сразу следует вложение $W(D, \Lambda) \subset W$. С другой стороны, любая конечная линейная комбинация элементов системы $E(\Lambda)$ представляет собой ряд (1), в котором отлично от нуля лишь конечное число коэффициентов. Поэтому $E(\Lambda) \subset W(D, \Lambda)$. Таким образом, верны также вложения

$$
\overline{E(\Lambda)} \subset \overline{W(D, \Lambda)} \subset \bar{W}
$$

Первое и последнее из этих множеств совпадают с $W$. Следовательно, мы имеем равенство $\overline{W(D, \Lambda)}=W$.

Проблема фундаментального принципа состоит в том, чтобы выяснить условия, когда $W$ совпадает с пространством функций $W(D, \Lambda)$. В силу последнего равенства эта проблема равносильна проблеме замкнутости множества сумм $W(D, \Lambda)$ в пространстве $H(D)$.

Мы хотим показать, что равенство $m(\Lambda)=0$ является необходимым условием замкнутости подпространства $W(D, \Lambda)$ в пространстве $H(D)$ в случае ограниченной выпуклой области $D$. Но прежде сделаем еще одно наблюдение.

Пусть $t>0$ и $D_{t}$ - область, полученная из $D$ при помощи преобразования гомотетии с центром в начале координат и коэффициентом $t$, т. е.

$$
D_{t}=\left\{z^{\prime}=t z: z \in D\right\} \text {. }
$$

Положим

$$
\Lambda(t)=\left\{t^{-1} \lambda_{k}, m_{k}\right\} .
$$

Очевидно, что при любом $t>0$ из равенств $\sigma(\Lambda)=m(\Lambda)=0$ следуют равенства $\sigma(\Lambda(t))=m(\Lambda(t))=0$ и наоборот. Кроме того, нетрудно показать, что имеет место следующий результат.

Лемма 1. Подпространства $W(D, \Lambda)$ u $W\left(D_{t}, \Lambda(t)\right)$ замкнуты или незамкнуты соответственно в $H(D)$ и в $H\left(D_{t}\right)$ одновременно.

Символами $B(z, r)$ и $S(z, r)$ будем обозначать соответственно открытый круг и окружность с центром в точке $z$ и радиуса $r$, а символом $\mathbb{S}$ - окружность $S(0,1)$. Пусть

$$
H_{M}(\lambda)=\sup _{z \in M} \operatorname{Re}(z \lambda), \quad \lambda \in \mathbb{C},
$$

- опорная функция множества $M \subset \mathbb{C}$ (точнее говоря, комплексно-сопряженного к $M$ множества). Она является выпуклой, положительно однородной порядка один и полунепрерывной снизу. Если $M$ ограничено, то функция $H_{M}(\lambda)$ непрерывна в комплексной плоскости (см. [16]).

Сформулируем и докажем, наконец, основной результат параграфа - необходимое условие замкнутости подпространства $W(D, \Lambda)$. 
Теорема 1. Пусть $D$ - ограниченная выпуклая область в $\mathbb{C}$ и последовательность $\Lambda=\left\{\lambda_{k}, m_{k}\right\}_{k=1}^{\infty}$ такова, что система $E(\Lambda)$ неполна в пространстве $H(D)$. Предположсим, что $W(D, \Lambda)$ - замкнутое подпространство в $H(D)$. Тогда $m(\Lambda)=0$.

Доказательство. Предположим, что утверждение теоремы неверно, т.е. $m(\Lambda)>0$. Отметим, что случай, когда $m(\Lambda)=\infty$, невозможен, так как $m(\Lambda)$, очевидно, не превосходит $N(\Lambda)$, а последняя величина, как уже отмечалось выше, конечна. Тогда, согласно нашему предположению, найдутся положительное число $\tau$ и подпоследовательность $\left\{\lambda_{k_{j}}, m_{k_{j}}\right\}$, такие, что последовательность $\left\{m_{k_{j}} /\left|\lambda_{k_{j}}\right|\right\}$ сходится к $\tau$, когда $j \rightarrow \infty$. Переходя еще раз к подпоследовательности, можно считать, что $\left\{\lambda_{k_{j}} /\left|\lambda_{k_{j}}\right|\right\}$ также сходится к некоторой точке $\xi$ окружности $\mathbb{S}$. Рассмотрим по отдельности три возможные ситуации: начало координат лежит во внешности области $D$, в самой области $D$ и на ее границе.

1) Пусть $0 \notin \bar{D}$. Поскольку $D-$ ограниченная область, согласно лемме 1 (делая, если необходимо, преобразование гомотетии с центром в нуле), можно считать, что для некоторой точки $a$ окружности $\mathbb{S}$ (очевидно, она является неединственной) круг $B(a, 1)$ содержит замыкание области $D$. Рассмотрим ряд

$$
\sum_{j=1}^{\infty} c_{j}(z-a)^{m(j)} \exp \left(\lambda_{k_{j}} z\right)
$$

где $m(j)=m_{k_{j}}-1, c_{j}=\exp \left(-H_{K}\left(\lambda_{k_{j}}\right)\right)$ и $K$ - некоторый выпуклый компакт из области $D$. Покажем, что при подходящем выборе компакта $K$ этот ряд сходится в топологии пространства $H(D)$. Для этого, прежде всего, заметим, что в силу включения $\bar{D} \subset B(a, 1)$ найдется радиус $r \in(0,1)$, такой, что круг $B(a, r)$ все еще содержит область $D$. Поэтому для каждого $z \in D$ верна оценка $\begin{aligned}\left|c_{j}(z-a)^{m(j)} \exp \left(\lambda_{k_{j}} z\right)\right| & \leqslant c_{j} r^{m(j)} \exp \left(\operatorname{Re}\left(\lambda_{k_{j}} z\right)\right) \leqslant r^{m(j)} \exp \left(\operatorname{Re}\left(\lambda_{k_{j}} z\right)-H_{K}\left(\lambda_{k_{j}}\right)\right) \\ & \leqslant r^{m(j)} \exp \left(H_{D}\left(\lambda_{k_{j}}\right)-H_{K}\left(\lambda_{k_{j}}\right)\right), \quad j=1,2, \ldots .\end{aligned}$

При получении последнего неравенства мы воспользовались определением опорной функции области $D$. Так как $\left\{m_{k_{j}} /\left|\lambda_{k_{j}}\right|\right\}$ сходится к $\tau$, когда $j \rightarrow \infty$, то для некоторого номера $j_{0}$ имеем

$$
m(j) \geqslant \tau\left|\lambda_{k_{j}}\right| / 2, \quad j \geqslant j_{0} .
$$

Следовательно, из предыдущего получаем

$$
\begin{aligned}
\left|c_{j}(z-a)^{m(j)} \exp \left(\lambda_{k_{j}} z\right)\right| & \leqslant \exp \left(m(j) \ln r+H_{D}\left(\lambda_{k_{j}}\right)-H_{K}\left(\lambda_{k_{j}}\right)\right) \\
& \leqslant \exp \left(-\beta\left|\lambda_{k_{j}}\right|+H_{D}\left(\lambda_{k_{j}}\right)-H_{K}\left(\lambda_{k_{j}}\right)\right), \quad j \geqslant j_{0},
\end{aligned}
$$

где $\beta=-2^{-1} \tau \ln r>0$. Выберем теперь компакт $K$ из области $D$, настолько большой, т. е. настолько «близкий» к самой области $D$, что выполнена оценка

$$
H_{D}(\lambda) \leqslant H_{K}(\lambda)+2^{-1} \beta|\lambda|, \quad \lambda \in \mathbb{C}
$$

(в силу ограниченности области $D$ такой компакт найдется). Отсюда и из предыдущего неравенства получаем

$$
\left|c_{j}(z-a)^{m(j)} \exp \left(\lambda_{k_{j}} z\right)\right| \leqslant \exp \left(-2^{-1} \beta\left|\lambda_{k_{j}}\right|\right), \quad j \geqslant j_{0} .
$$


Тогда с учетом сделанного в начале параграфа замечания о том, что в условиях теоремы верно равенство $\sigma(\Lambda)=0$, для всех $z \in D$ имеем

$$
\sum_{j=j_{0}}^{\infty}\left|c_{j}(z-a)^{m(j)} \exp \left(\lambda_{k_{j}} z\right)\right| \leqslant \sum_{j=j_{0}}^{\infty} \exp \left(-2^{-1} \beta\left|\lambda_{k_{j}}\right|\right)<\infty .
$$

Это означает, что ряд (2) сходится равномерно во всей области $D$. Поэтому функция

$$
g(z)=\sum_{j=1}^{\infty} c_{j}(z-a)^{m(j)} \exp \left(\lambda_{k_{j}} z\right)
$$

аналитична в $D$. По условию подпространство $W(D, \Lambda)$ замкнуто в $H(D)$. Следовательно, функция $g(z)$ как предел элементов подпространства $W(D, \Lambda)$ (частичных сумм ряда $(2))$ принадлежит $W(D, \Lambda)$. Поэтому, согласно определению $W(D, \Lambda)$, имеет место представление

$$
g(z)=\sum_{k=1, n=0}^{\infty, m_{k}-1} d_{k, n} z^{n} \exp \left(\lambda_{k} z\right), \quad z \in D,
$$

причем последний ряд сходится в топологии пространства $H(D)$.

Пусть $\left\{\mu_{k, n}\right\}_{k=1, n=0}^{\infty, m_{k}-1}-$ биортогональная к семейству $E(\Lambda)$ последовательность функционалов из сопряженного пространства $H^{*}(D)$, о существовании которой говорилось выше. Тогда в силу линейности и непрерывности этих функционалов из (4) получаем

$$
\mu_{k_{j}, 0}(g)=\sum_{k=1, n=0}^{\infty, m_{k}-1} d_{k, n} \mu_{k_{j}, 0}\left(z^{n} \exp \left(\lambda_{k} z\right)\right)=d_{k_{j}, 0}, \quad j=1,2, \ldots
$$

С другой стороны, в силу представления (3) имеем

$\mu_{k_{j}, 0}(g(z))=\sum_{j=1}^{\infty} c_{j} \mu_{k_{j}, 0}\left((z-a)^{m(j)} \exp \left(\lambda_{k_{j}} z\right)\right)=(-1)^{m(j)} c_{j} a^{m(j)}, \quad j=1,2, \ldots$

Поэтому $\left|d_{k_{j}, 0}\right|=\left|c_{j} a^{m(j)}\right|=c_{j}, j=1,2, \ldots$, так как точка $a$ лежит на окружности $\mathbb{S}$. Поскольку $K-$ компакт в $D$, существует точка $z_{0} \in D$, такая, что

$$
\operatorname{Re}\left(z_{0} \xi\right)>H_{K}(\xi)
$$

Тогда из сходимости последовательности $\left\{\lambda_{k_{j}} /\left|\lambda_{k_{j}}\right|\right\}$ к точке $\xi$ и непрерывности и однородности опорной функции компакта для всех достаточно больших номеров $j$ следует соотношение

$$
\operatorname{Re}\left(z_{0} \lambda_{k_{j}}\right)>H_{K}\left(\lambda_{k_{j}}\right) .
$$

Отсюда для этих же $j$ с учетом определения коэффициентов $c_{j}$ получаем

$$
\left|d_{k_{j}, 0} \exp \left(\lambda_{k_{j}} z_{0}\right)\right|=\left|c_{j} \exp \left(\lambda_{k_{j}} z_{0}\right)\right|=\exp \left(\operatorname{Re}\left(\lambda_{k_{j}} z_{0}\right)-H_{K}\left(\lambda_{k_{j}}\right)\right)>1 .
$$

Это противоречит необходимому условию сходимости ряда (4) в точке $z_{0}$. Таким образом, предположение о том, что $m(\Lambda) \neq 0$, неверно. Следовательно, в этом случае теорема доказана. 
2) Пусть теперь $0 \in D$. Через $T$ обозначим пересечение опорной прямой области $D$ в направлении $\xi$ с границей этой области, т. е.

$$
T=\left\{z: \operatorname{Re}(z \xi)=H_{D}(\xi)\right\} \cap \partial D .
$$

В силу ограниченности области $D$ множество $T$ непусто и является точкой или отрезком. Как и выше (делая, если это необходимо, преобразование гомотетии с центром в нуле), можно считать, что для некоторой точки $a$ окружности $\mathbb{S}$ круг $B(a, 1)$ содержит множество $T$ и, кроме того, область $D$ лежит в круге $B(0,1)$.

Рассмотрим ряд

$$
\sum_{j=1}^{\infty} c_{j}(z-a)^{n(j)} \exp \left(\lambda_{k_{j}} z\right),
$$

где $c_{j}=\exp \left(-H_{K}\left(\lambda_{k_{j}}\right)\right), j=1,2, \ldots, K$ - некоторый выпуклый компакт в $D$ и $\{n(j)\}$ - последовательность натуральных чисел. Покажем, что при подходящем выборе $K$ и чисел $n(j), j=1,2, \ldots$, этот ряд сходится в топологии пространства $H(D)$. Для этого, прежде всего, заметим, что $T$ - компакт, а потому лежит в круге $B(a, 1)$ вместе с некоторой своей окрестностью. Следовательно, найдется $\varepsilon>0$, такое, что множество

$$
T(\varepsilon)=\left\{z: \operatorname{Re}(z \xi) \geqslant H_{D}(\xi)-\varepsilon\right\} \cap D
$$

компактно и лежит в $B(a, 1)$. Другими словами, $T(\varepsilon)$ содержится в круге $B(a, r)$ при некотором $r$ из интервала $\left(e^{-1}, 1\right)$.

Поскольку последовательность $\left\{m_{k_{j}} /\left|\lambda_{k_{j}}\right|\right\}$ сходится к $\tau$, когда $j \rightarrow \infty$, для каждого $j$, начиная с некоторого номера $j_{0}$, мы можем выбрать натуральное число $n(j)$, которое не превосходит $m(j)$ и, кроме этого, удовлетворяет неравенствам

$$
\gamma\left|\lambda_{k_{j}}\right| \leqslant n(j) \leqslant \varepsilon\left|\lambda_{k_{j}}\right| / 2,
$$

где $\gamma=\min \{\varepsilon / 4, \tau / 2\}$. Выберем теперь компакт $K$ из области $D$, настолько большой, что выполнена оценка

$$
H_{D}(\lambda) \leqslant H_{K}(\lambda)-2^{-1} \gamma|\lambda| \ln r, \quad \lambda \in \mathbb{C}
$$

(это можно сделать, так как $\ln r<0$ ). Учитывая вложение $T(\varepsilon) \subset B(a, r)$, определение коэффициентов $c_{j}$ и опорной функции области $D$, из $(6)$ и $(7)$ получаем

$$
\begin{aligned}
\left|c_{j}(z-a)^{n(j)} \exp \left(\lambda_{k_{j}} z\right)\right| & \leqslant \exp \left(n(j) \ln r+H_{D}\left(\lambda_{k_{j}}\right)-H_{K}\left(\lambda_{k_{j}}\right)\right) \\
& \leqslant \exp \left(\gamma\left|\lambda_{k_{j}}\right| \ln r+H_{D}\left(\lambda_{k_{j}}\right)-H_{K}\left(\lambda_{k_{j}}\right)\right) \\
& \leqslant \exp \left(2^{-1} \gamma\left|\lambda_{k_{j}}\right| \ln r\right), \quad j \geqslant j_{0}, \quad z \in T(\varepsilon) .
\end{aligned}
$$

Так как последовательность $\left\{\lambda_{k_{j}} /\left|\lambda_{k_{j}}\right|\right\}$ сходится к точке $\xi$ при $j \rightarrow \infty$, то, увеличивая при необходимости номер $j_{0}$, можно считать, что для $j \geqslant j_{0}$ верна оценка

$$
\left|\operatorname{Re}\left(z\left(\xi-\lambda_{k_{j}} /\left|\lambda_{k_{j}}\right|\right)\right)\right| \leqslant \varepsilon / 8 \quad \text { для любого } z,|z| \leqslant 1 .
$$

В частности, эта оценка имеет место для всех $z \in D \subset B(0,1)$. Следовательно,

$$
\operatorname{Re}\left(z \lambda_{k_{j}}\right) \leqslant\left|\lambda_{k_{j}}\right| \operatorname{Re}(z \xi)+16^{-1} \varepsilon\left|\lambda_{k_{j}}\right|, \quad z \in D .
$$


Кроме того, используя непрерывность опорной функции ограниченной области, можно также считать, что

$$
\left|\lambda_{k_{j}}\right| H_{D}(\xi) \leqslant H_{D}\left(\lambda_{k_{j}}\right)+16^{-1} \varepsilon\left|\lambda_{k_{j}}\right|, \quad j \geqslant j_{0} .
$$

Пусть теперь $z \in D \backslash T(\varepsilon)$. Тогда, учитывая определение коэффициентов $c_{j}$, вложение $D \subset B(0,1)$ (в силу которого $|z-a| \leqslant 2, z \in D$ ) и неравенство (9), имеем

$$
\begin{aligned}
\left|c_{j}(z-a)^{n(j)} \exp \left(\lambda_{k_{j}} z\right)\right| & \leqslant \exp \left(n(j) \ln |z-a|+\operatorname{Re}\left(z \lambda_{k_{j}}\right)-H_{K}\left(\lambda_{k_{j}}\right)\right) \\
& \leqslant \exp \left(n(j) \ln 2+\left|\lambda_{k_{j}}\right| \operatorname{Re}(z \xi)+16^{-1} \varepsilon\left|\lambda_{k_{j}}\right|-H_{K}\left(\lambda_{k_{j}}\right)\right) \\
& \leqslant \exp \left(n(j)+\left|\lambda_{k_{j}}\right| \operatorname{Re}(z \xi)+16^{-1} \varepsilon\left|\lambda_{k_{j}}\right|-H_{K}\left(\lambda_{k_{j}}\right)\right) .
\end{aligned}
$$

Отсюда и из определения множества $T(\varepsilon)$ для всех $z \in D \backslash T(\varepsilon)$ получаем

$$
\begin{aligned}
& \left|c_{j}(z-a)^{n(j)} \exp \left(\lambda_{k_{j}} z\right)\right| \\
& \quad \leqslant \exp \left(n(j)+\left|\lambda_{k_{j}}\right|\left(H_{D}(\xi)-\varepsilon\right)+16^{-1} \varepsilon\left|\lambda_{k_{j}}\right|-H_{K}\left(\lambda_{k_{j}}\right)\right), \quad z \in D \backslash T(\varepsilon) .
\end{aligned}
$$

Это вместе с (10) дает нам неравенство

$$
\begin{aligned}
& \left|c_{j}(z-a)^{n(j)} \exp \left(\lambda_{k_{j}} z\right)\right| \\
& \leqslant \exp \left(n(j)-\varepsilon\left|\lambda_{k_{j}}\right|+H_{D}\left(\lambda_{k_{j}}\right)+8^{-1} \varepsilon\left|\lambda_{k_{j}}\right|-H_{K}\left(\lambda_{k_{j}}\right)\right), \quad j \geqslant j_{0}, z \in D \backslash T(\varepsilon) .
\end{aligned}
$$

Отсюда с учетом (6), (7) и неравенств $0>\ln r>-1$ (которые выполнены в силу выбора числа $r$ ) получаем

$$
\begin{aligned}
\left|c_{j}(z-a)^{n(j)} \exp \left(\lambda_{k_{j}} z\right)\right| & \leqslant \exp \left(\frac{\varepsilon\left|\lambda_{k_{j}}\right|}{2}-\varepsilon\left|\lambda_{k_{j}}\right|+8^{-1} \varepsilon\left|\lambda_{k_{j}}\right|-2^{-1} \gamma\left|\lambda_{k_{j}}\right| \ln r\right) \\
& \leqslant \exp \left(\frac{-3 \varepsilon\left|\lambda_{k_{j}}\right|}{8}-\frac{\gamma\left|\lambda_{k_{j}}\right| \ln r}{2}\right) \\
& \leqslant \exp \left(\frac{-3 \varepsilon\left|\lambda_{k_{j}}\right|}{8}+\frac{\gamma\left|\lambda_{k_{j}}\right|}{2}\right), \quad z \in D \backslash T(\varepsilon), j \geqslant j_{0} .
\end{aligned}
$$

Вспоминая теперь определение числа $\gamma$, окончательно имеем

$$
\left|c_{j}(z-a)^{n(j)} \exp \left(\lambda_{k_{j}} z\right)\right| \leqslant \exp \left(-\varepsilon\left|\lambda_{k_{j}}\right| / 4\right), \quad z \in D \backslash T(\varepsilon), j \geqslant j_{0} .
$$

Отсюда и из (8) для всех $z \in D$ и $j \geqslant j_{0}$ получаем неравенство

$$
\left|c_{j}(z-a)^{n(j)} \exp \left(\lambda_{k_{j}} z\right)\right| \leqslant \exp \left(-\rho\left|\lambda_{k_{j}}\right|\right),
$$

где

$$
\rho=\min \{\varepsilon / 4,-(\gamma \ln r) / 2\} .
$$

Таким образом, как и в случае 1$)$, ряд (5) сходится равномерно в области $D$ (а значит, и в топологии пространства $H(D)$ ) к некоторой функции $g$, аналитической в $D$. Все дальнейшие рассуждения практически дословно (нужно лишь заменить числа $m(j)$ на $n(j))$ повторяют соответствующие рассуждения в случае 1).

Следовательно, равенство $m(\Lambda)=0$ имеет место и в случае 2 ).

3) Пусть, наконец, $0 \in \partial D$. В этом случае некоторая опорная прямая области $D$, которую мы обозначим символом $l$, проходит через начало координат (если 
таких прямых несколько, то в качестве $l$ выберем любую из них). Через $a$ обозначим одну из точек пересечения прямой $l$ и окружности $\mathbb{S}$. Тогда $-a$ является другой точкой их пересечения. Прямая $l$ делит плоскость на две полуплоскости. Область $D$ целиком лежит в одной из этих полуплоскостей. Точку окружности $\mathbb{S}$, которая принадлежит той же полуплоскости, что и $D$, и лежит на прямой $l^{\prime}$, перпендикулярной к $l$ и проходящей через начало координат, обозначим через $b$. Прямая $l$ делит круг $B(0,1 / 2)$ на два полукруга. Делая преобразование гомотетии с центром в нуле, можно считать, что область $D$ лежит в одном из этих полукругов, который обозначим через $B^{\prime}$ (в том, который лежит по ту же сторону от прямой $l$, что и точка $b$ ).

Поскольку последовательность $\left\{m_{k_{j}} /\left|\lambda_{k_{j}}\right|\right\}_{j=1}^{\infty}$ сходится к числу $\tau$, отличному от нуля, переходя к подпоследовательности, можно также считать, что выполнены неравенства

$$
2 \tau\left|\lambda_{k_{j}}\right| \geqslant m(j) \geqslant 2 \nu(j) \geqslant 4 \nu\left|\lambda_{k_{j}}\right|,
$$

где $\nu>0$ и $\nu(j), j=1,2, \ldots,-$ любые натуральные числа, удовлетворяющие этим неравенствам. Рассмотрим ряд

$$
\sum_{j=1}^{\infty} c_{j} \exp \left(\delta\left|\lambda_{k_{j}}\right|\right)\left(z\left(z^{2}-a^{2}\right)(z-b)\right)^{\nu(j)} \exp \left(\lambda_{k_{j}} z\right),
$$

где

$$
c_{j}=\exp \left(-\sup _{z \in D}\left(\nu(j) \ln |z|+\operatorname{Re}\left(z \lambda_{k_{j}}\right)\right)\right) .
$$

Покажем, что при подходящем выборе числа $\delta>0$ ряд (11) сходится в топологии пространства $H(D)$. Для этого, прежде всего, оценим модуль многочлена $p(z)=\left(z^{2}-a^{2}\right)(z-b)$ на границе полукруга $B^{\prime}$. Пусть $z \in[-a, a]$ и $x=|z|$. Рассмотрим систему координат, образованную прямыми $l$ и $l^{\prime}$. Меняя, если это необходимо, местами точки $a$ и $-a$, можно считать, что в этой системе координат точки $a$ и $b$ имеют соответственно координаты $(1,0)$ и $(0,1)$. Тогда легко убеждаемся, что

$$
|p(z)|=\left(1-x^{2}\right) \sqrt{1+x^{2}}, \quad z \in[-a, a] .
$$

Непосредственно проверяем, что функция, стоящая в правой части этого равенства, строго убывает на отрезке $[0,1]$ и равна единице при $x=0$. Следовательно, верны соотношения

$$
|p(z)|<1, \quad z \in[-a, a] \backslash\{0\}, \quad|p(0)|=1 .
$$

Пусть теперь $z$ принадлежит той части границы полукруга $B^{\prime}$, которая является полуокружностью. Через $x$ обозначим расстояние от точки $z$ до прямой $l^{\prime}$, а через $y$ - расстояние от точки $z$ до прямой $l$. Другими словами, $(x, y)-$ это координаты точки $z$ в указанной выше системе координат. Тогда прямым подсчетом получаем

$$
|p(z)|=\sqrt{(1-x)^{2}+y^{2}} \sqrt{(1+x)^{2}+y^{2}} \sqrt{(1-y)^{2}+x^{2}} .
$$


Раскрывая под радикалами скобки и замечая, что $x^{2}+y^{2}=1 / 4$ (так как точка $z$ лежит на окружности $S(0,1 / 2))$, имеем

$$
\begin{aligned}
|p(z)| & =\sqrt{5 / 4-2 x} \sqrt{5 / 4+2 x} \sqrt{5 / 4-2 y} \\
& =\sqrt{25 / 16-4 x^{2}} \sqrt{5 / 4-2 y}=\sqrt{9 / 16+4 y^{2}} \sqrt{5 / 4-2 y} .
\end{aligned}
$$

Нетрудно показать, что последняя функция строго убывает при $y \in[0,1 / 2]$, причем на концах этого отрезка она принимает значения, строго меньшие единицы. Таким образом, на всей рассматриваемой полуокружности верна оценка $|p(z)|<1$. Следовательно, с учетом (12) получаем

$$
|p(z)|<1, \quad z \in \partial B^{\prime} \backslash\{0\}, \quad|p(0)|=1 .
$$

Отсюда и из принципа максимума модуля для аналитических функций следует оценка

$$
|p(z)|<1, \quad z \in \overline{B^{\prime}} \backslash\{0\} .
$$

Фиксируем какую-нибудь точку $z_{0}$ области $D$. Тогда с учетом определения чисел $c_{j}, j=1,2, \ldots$, верны неравенства

$$
\begin{gathered}
c_{j}|z|^{\nu(j)} \exp \left(\operatorname{Re}\left(\lambda_{k_{j}} z\right)\right) \leqslant \exp \left(-\nu(j) \ln \left|z_{0}\right|-\operatorname{Re}\left(z_{0} \lambda_{k_{j}}\right)\right)|z|^{\nu(j)} \exp \left(\operatorname{Re}\left(\lambda_{k_{j}} z\right)\right) \\
=\exp \left(\nu(j)\left(\ln |z|-\ln \left|z_{0}\right|\right)+\operatorname{Re}\left(z \lambda_{k_{j}}\right)-\operatorname{Re}\left(z_{0} \lambda_{k_{j}}\right)\right), \quad j=1,2, \ldots .
\end{gathered}
$$

Учитывая, что $\nu\left|\lambda_{k_{j}}\right| \leqslant \nu(j) \leqslant \tau\left|\lambda_{k_{j}}\right|, j=1,2, \ldots$, получаем отсюда для всех $z$ из круга $B(0, r)$, где $r \in(0,1)$,

$$
\begin{aligned}
& c_{j}|z|^{\nu(j)} \exp \left(\operatorname{Re}\left(\lambda_{k_{j}} z\right)\right) \leqslant \exp \left(\nu\left|\lambda_{k_{j}}\right| \ln r+\tau\left|\lambda_{k_{j}}\right||\ln | z_{0}||+\left(|z|+\left|z_{0}\right|\right)\left|\lambda_{k_{j}}\right|\right) \\
& \leqslant \exp \left(\nu\left|\lambda_{k_{j}}\right| \ln r+\tau\left|\lambda_{k_{j}}\right||\ln | z_{0}||+\left(1+\left|z_{0}\right|\right)\left|\lambda_{k_{j}}\right|\right), \quad j=1,2, \ldots .
\end{aligned}
$$

Выберем $r_{0} \in(0,1)$ настолько маленьким, что выполнено неравенство

$$
\nu \ln r_{0}+\tau|\ln | z_{0}||+\left(1+\left|z_{0}\right|\right)<-1 .
$$

Тогда из предыдущего получаем, что

$$
c_{j}|z|^{\nu(j)} \exp \left(\operatorname{Re}\left(\lambda_{k_{j}} z\right)\right) \leqslant \exp \left(-\left|\lambda_{k_{j}}\right|\right), \quad|z|<r_{0}, j=1,2, \ldots
$$

Так как область $D$ лежит в полукруге $B^{\prime}$, а $\bar{D} \backslash B\left(0, r_{0}\right)$ является компактом, то, согласно (13),

$$
\max _{z \in \bar{D} \backslash B\left(0, r_{0}\right)}|p(z)|<1 .
$$

Следовательно, с учетом того, что $\nu\left|\lambda_{k_{j}}\right| \leqslant \nu(j), j=1,2, \ldots$, найдется $\varepsilon \in(0,1)$, для которого имеет место оценка

$$
|p(z)|^{\nu(j)} \leqslant \exp \left(-\varepsilon\left|\lambda_{k_{j}}\right|\right), \quad z \in \bar{D} \backslash B\left(0, r_{0}\right), j=1,2, \ldots .
$$

Выберем теперь в качестве $\delta$ какое-нибудь число из интервала $(0, \varepsilon / 2)$. Тогда из (13) и (14) следует неравенство

$$
\begin{aligned}
& c_{j} \exp \left(\delta\left|\lambda_{k_{j}}\right|\right)|z|^{\nu(j)}|p(z)|^{\nu(j)} \exp \left(\operatorname{Re}\left(\lambda_{k_{j}} z\right)\right) \\
& \quad \leqslant \exp \left(-\left|\lambda_{k_{j}}\right|\right) \exp \left(\varepsilon\left|\lambda_{k_{k}}\right| / 2\right) \leqslant \exp \left(-\varepsilon\left|\lambda_{k_{j}}\right| / 2\right),
\end{aligned}
$$


где $z \in \bar{D} \cap B\left(0, r_{0}\right), j=1,2, \ldots$ Кроме того, в силу (15) и определения чисел $c_{j}$ получаем

$$
\begin{aligned}
& c_{j} \exp \left(\delta\left|\lambda_{k_{j}}\right|\right)|z|^{\nu(j)}|p(z)|^{\nu(j)} \exp \left(\operatorname{Re}\left(\lambda_{k_{j}} z\right)\right) \\
& \quad \leqslant \exp \left(-\sup _{z \in D}\left(\nu(j) \ln |z|+\operatorname{Re}\left(\lambda_{k_{j}} z\right)\right)\right) \exp \left(\varepsilon\left|\lambda_{k_{j}}\right| / 2-\varepsilon\left|\lambda_{k_{j}}\right|+\operatorname{Re}\left(\lambda_{k_{j}} z\right)\right)|z|^{\nu(j)} \\
& \quad=\exp \left(-\sup _{z \in D}\left(\nu(j) \ln |z|+\operatorname{Re}\left(\lambda_{k_{j}} z\right)\right)+\operatorname{Re}\left(\lambda_{k_{j}} z\right)+\nu(j) \ln |z|-\varepsilon\left|\lambda_{k_{j}}\right| / 2\right) \\
& \quad \leqslant \exp \left(-\varepsilon\left|\lambda_{k_{j}}\right| / 2\right), \quad z \in \bar{D} \cap B\left(0, r_{0}\right), j=1,2, \ldots
\end{aligned}
$$

Объединяя эту и предыдущую оценку, имеем

$$
\sum_{j=1}^{\infty} c_{j} \exp \left(\delta\left|\lambda_{k_{j}}\right|\right)|z|^{\nu(j)}|p(z)|^{\nu(j)} \exp \left(\operatorname{Re}\left(\lambda_{k_{j}} z\right)\right) \leqslant \sum_{j=1}^{\infty} \exp \left(-\varepsilon\left|\lambda_{k_{j}}\right| / 2\right), \quad z \in \bar{D} .
$$

Так же, как и выше, по лемме 1 из работы [17] получаем, что последний ряд сходится. Таким образом, ряд (11) сходится равномерно во всей области $D$. Следовательно, его сумма $g(z)$ является функцией, аналитической в $D$.

С другой стороны, как и в случае 1), имеет место представление (4) (здесь мы учитываем неравенство $\left.m_{k_{j}}-1=m(j) \geqslant 4 \nu(j)\right)$. При этом, как нетрудно убедиться,

$$
\left|d_{k_{j}, \nu(j)}\right|=\left|\mu_{k_{j}, \nu(j)}(g)\right|=\left|\exp \left(\delta\left|\lambda_{k_{j}}\right|\right) c_{j}\left(a^{2} b\right)^{\nu(j)}\right|=c_{j} \exp \left(\delta\left|\lambda_{k_{j}}\right|\right)
$$

для всех $j=1,2, \ldots$

Поскольку $D$ ограничена, для каждого $j=1,2, \ldots$ найдется точка $z_{j} \in \bar{D}$, такая, что

$$
\left|z_{j}\right|^{\nu(j)} \exp \left(\operatorname{Re}\left(z_{j} \lambda_{k_{j}}\right)\right)=\sup _{z \in D}\left(|z|^{\nu(j)} \exp \left(\operatorname{Re}\left(z \lambda_{k_{j}}\right)\right)\right)=c_{j}^{-1} .
$$

Выберем подпоследовательность натуральных чисел $j(1), j(2), \ldots$ так, что последовательность $\left\{z_{j(p)}\right\}_{p=1}^{\infty}$ сходится к некоторой точке $\tilde{z} \in \bar{D}$. В силу неравенства (14) точки $z_{j}, j=1,2, \ldots$, а вместе с ними и точка $\tilde{z}$ не попадают в круг $B\left(0, r_{0}\right)$.

Фиксируем $\delta^{\prime} \in(0, \delta / 4)$, удовлетворяющее условию

$$
\tau \ln \left(1+4 \delta^{\prime} / r_{0}\right) \leqslant \delta / 2 .
$$

Пусть $z^{\prime} \in D \cap B\left(\tilde{z}, \delta^{\prime}\right)$. Очевидно, можно считать, что $\left|z^{\prime}\right| \geqslant r_{0} / 2$. Поскольку последовательность $\left\{z_{j(p)}\right\}_{p=1}^{\infty}$ сходится к $\tilde{z}$, для всех номеров $p$, начиная с некоторого $p_{0}$, выполнено неравенство

$$
\left|z^{\prime}-z_{j(p)}\right| \leqslant 2 \delta^{\prime}, \quad p \geqslant p_{0} .
$$

Тогда, учитывая еще неравенства $\left|z^{\prime}\right| \geqslant r_{0} / 2, \nu(j) \leqslant \tau\left|\lambda_{k_{j}}\right|, j=1,2, \ldots$, выбор 
точек $z_{j}$ и числа $\delta^{\prime}$ имеем

$$
\begin{aligned}
c_{j(p)}^{-1} & =\left|z_{j(p)}\right|^{\nu(j(p))} \exp \left(\operatorname{Re}\left(\lambda_{k_{j(p)}} z_{j(p)}\right)\right) \\
& =\left|\left(z_{j(p)}-z^{\prime}\right)+z^{\prime}\right|^{\nu(j(p))} \exp \left(\operatorname{Re}\left(\left(z_{j(p)}-z^{\prime}+z^{\prime}\right) \lambda_{k_{j(p)}}\right)\right) \\
& \leqslant \exp \left(\nu(j(p)) \ln \left|z_{j(p)}-z^{\prime}+z^{\prime}\right|+\operatorname{Re}\left(\left(z_{j(p)}-z^{\prime}+z^{\prime}\right) \lambda_{\left.k_{j(p)}\right)}\right)\right. \\
& \leqslant \exp \left(\nu(j(p))\left(\ln \left|z^{\prime}\right|+\ln \left(1+\frac{\left|z_{j(p)}-z^{\prime}\right|}{\left|z^{\prime}\right|}\right)\right)\right. \\
& \leqslant \exp \left(\nu(j(p))\left(\ln \left|z^{\prime}\right|+\ln \left(1+\frac{\left.\left.2 \delta_{k_{j(p)}}\right)+\left|z_{j(p)}-z^{\prime}\right|\left|\lambda_{k_{j(p)}}\right|\right)}{\left|z^{\prime}\right|}\right)+\operatorname{Re}\left(\lambda_{k_{j(p)}} z^{\prime}\right)+2 \delta^{\prime}\left|\lambda_{k_{j(p)}}\right|\right)\right. \\
& \leqslant \exp \left(\nu(j(p))\left(\ln \left|z^{\prime}\right|+\ln \left(1+\frac{4 \delta^{\prime}}{r_{0}}\right)\right)+\operatorname{Re}\left(\lambda_{k_{j(p)}} z^{\prime}\right)+\frac{\delta^{\prime}\left|\lambda_{k_{j(p)}}\right|}{2}\right) \\
& \leqslant \exp \left(\nu(j(p))\left(\ln \left|z^{\prime}\right|+\frac{\delta}{2 \tau}\right)+\operatorname{Re}\left(\lambda_{k_{j(p)}} z^{\prime}\right)+\frac{\delta^{\prime}\left|\lambda_{k_{j(p)}}\right|}{2}\right) \\
& \leqslant \exp \left(\nu(j(p)) \ln \left|z^{\prime}\right|+\frac{\delta\left|\lambda_{k_{j(p)}}\right|}{2}+\operatorname{Re}\left(\lambda_{k_{j(p)}} z^{\prime}\right)+\frac{\delta^{\prime}\left|\lambda_{k_{j(p)}}\right|}{2}\right) \\
& =\left|z^{\prime}\right| \nu(j(p)) \exp \left(\operatorname{Re}\left(z^{\prime} \lambda_{k_{j(p)}}\right)+\delta\left|\lambda_{k_{j(p)}}\right|\right), \quad p \frac{p}{p_{0} .}
\end{aligned}
$$

Следовательно, с учетом (16) получаем

$$
\begin{aligned}
& \left|d_{k_{j(p)}, \nu(j(p))}\left(z^{\prime}\right)^{\nu(j(p))} \exp \left(z^{\prime} \lambda_{k_{j(p)}}\right)\right| \\
& \quad=\left|c_{j(p)}\left(z^{\prime}\right)^{\nu(j(p))} \exp \left(\delta\left|\lambda_{k_{j(p)}}\right|\right) \exp \left(\lambda_{k_{j(p)}} z^{\prime}\right)\right| \geqslant 1, \quad p \geqslant p_{0} .
\end{aligned}
$$

Это противоречит необходимому условию сходимости ряда $(4)$ в точке $z^{\prime} \in D$.

Таким образом, равенство $m(\Lambda)=0$ имеет место и в этом случае. Теорема полностью доказана.

Замечание. При разборе первого случая мы показали, что в области $D-a$ (сдвиг области $D)$ функция $g^{\prime}(w)=g(w+a)$, где $w=z-a$ и $g(z)$ определена по формуле (3), разлагается в ряд по системе $\left\{w^{n} \exp \left(\lambda_{k} w\right)\right\}_{k=1, n=0}^{\infty, m_{k}-1}$. В то же время функция $g(z)$ в области $D$ не разлагается в ряд по системе $\left\{z^{n} \exp \left(\lambda_{k} z\right)\right\}_{k=1, n=0}^{\infty, m_{k}-1}$. Это означает, что в случае, когда $m(\Lambda)>0$, эта система не сохраняет свойство базисности при сдвигах. Более того, можно показать, что условие $m(\Lambda)=0$ необходимо и достаточно для того, чтобы указанная система сохраняла свойство базисности при любом сдвиге.

\section{§3. Фундаментальный принцип}

В этом заключительном параграфе мы сформулируем и докажем справедливость фундаментального принципа для инвариантных подпространств, допускающих спектральный синтез. Как отмечалось во введении, этот результат уже был получен ранее в работе [4] при одном ограничении на кратность показателей ряда $(1): m(\Lambda)=0$. Здесь это ограничение устраняется.

Прежде чем привести указанный результат, введем еще некоторые обозначения. Для выпуклой области $D$ через $K(D)$ обозначим последовательность 
$\left\{K_{p}\right\}_{p=1}^{\infty}$ выпуклых компактов из области $D$, которая строго исчерпывает еe, т. е. $K_{p} \subset \operatorname{int} K_{p+1}, p=1,2, \ldots$ (здесь символом int обозначена внутренность множества), и $D=\bigcup_{p=1}^{\infty} K_{p}$.

Пусть $d=\left\{d_{k, n}\right\}_{k=1, n=0}^{\infty, m_{k}-1}-$ последовательность комплексных чисел. Для каждого $p=1,2, \ldots$ введем банахово пространство

$$
Q_{p}=\left\{d=\left\{d_{k, n}\right\}:\|d\|_{p}=\sup _{k, n}\left|d_{k, n}\right| \exp H_{K_{p}}\left(\lambda_{k}\right)<\infty\right\},
$$

где $K_{p} \in K(D)$. Пусть $Q(D)=\bigcap_{p} Q_{p}$. В пространстве $Q(D)$ определим метрику

$$
\rho\left(d, d^{\prime}\right)=\sum_{p=1}^{\infty} 2^{-p} \frac{\left\|d-d^{\prime}\right\|_{p}}{1+\left\|d-d^{\prime}\right\|_{p}} .
$$

С этой метрикой $Q(D)$ становится, очевидно, пространством Фреше. Отметим, что если область $D^{\prime}$ содержит $D$, то множество $Q\left(D^{\prime}\right)$ является подмножеством в $Q(D)$.

Пусть $T$ - множество в комплексной плоскости и $\Theta-$ замкнутое подмножество единичной окружности с центром в начале координат. Положим

$$
T(\Theta)=\left\{z \in \mathbb{C}: \operatorname{Re}(z \xi)<H_{T}(\xi) \text { для любого } \xi \in \Theta\right\} \text {. }
$$

Нетрудно заметить, что $T(\Theta)$ является выпуклой областью. Для последовательности $\Lambda=\left\{\lambda_{k}, m_{k}\right\}$ через $\Theta(\Lambda)$ обозначим множество всех возможных пределов подпоследовательностей $\left\{\lambda_{k_{j}} /\left|\lambda_{k_{j}}\right|\right\}$. Очевидно, что $\Theta(\Lambda)$ является замкнутым подмножеством единичной окружности. Нам понадобится следующий результат из работы [17], который служит аналогом теоремы Абеля для рядов экспоненциальных мономов.

Теорема 2. Пусть последовательность $\Lambda$ такова, что $\sigma(\Lambda)=m(\Lambda)=0$. Предположим, что общий член ряда (1) ограничен на множестве $T \subset \mathbb{C}$. Кроме того, если начало координат является изолированной точкой множества T, то ограничена также последовательность $\left\{d_{k, n}\right\}_{k=1, n=0}^{\infty, m_{k}-1}$. Тогда $d=$ $\left\{d_{k, n}\right\} \in Q(D)$, где $D=T(\Theta(\Lambda))$, и для каждого $p=1,2, \ldots$ найдется постоянная $C_{p}>0$ (не зависящая от последовательности $d$ ), такая, что

$$
\sum_{k=1, n=0}^{\infty, m_{k}-1}\left|d_{k, n}\right| \sup _{z \in K_{p}}\left|z^{n} \exp \left(z \lambda_{k}\right)\right| \leqslant C_{p}\|d\|_{p+2} .
$$

В частности, ряд (1) сходится абсолютно и равномерно на любом компакте из области $D$.

Пусть $f(\lambda)$ - целая функция экспоненциального типа. Ее индикатором (верхним индикатором) называется функция

$$
h_{f}(\lambda)=\varlimsup_{t \rightarrow \infty} \frac{\ln |f(t \lambda)|}{t}, \quad \lambda \in \mathbb{C} .
$$

Символом $\underline{h}_{f}$ обозначим нижний индикатор функции $f$,

$$
\underline{h}_{f}(\lambda)=\lim _{\delta \rightarrow 0} \underline{\lim }_{t \rightarrow \infty} \frac{1}{\pi \delta^{2}} \int_{B(\lambda, \delta)} \frac{\ln |f(t z)|}{t} d x d y, \quad z=x+i y .
$$


Из определений индикаторов вытекает неравенство

$$
\underline{h}_{f}(\lambda) \leqslant h_{f}(\lambda), \quad \lambda \in \mathbb{C} .
$$

Говорят, что функция $f$ имеет (вполне) регулярный рост, если верно равенство [21, гл. 4, определение 4.1]

$$
\underline{h}_{f}=h_{f}(\lambda), \quad \lambda \in \mathbb{C} .
$$

Последовательность $\Lambda=\left\{\lambda_{k}, m_{k}\right\}_{k=1}^{\infty}$ называется (см. [22, гл. 2, §1]) правильно распределенным множеством при порядке один, если существует

$$
\lim _{r \rightarrow \infty} \sum_{\left|\lambda_{k}\right|<r} \frac{m_{k}}{\lambda_{k}}
$$

и $\Lambda$ имеет угловую плотность при порядке один, т. е. для всех открытых углов $T$ с вершиной в начале координат (за исключением, быть может, счетного их числа) существует $\lim _{r \rightarrow \infty} n(T, r) / r$, где $n(T, r)$ - число точек $\lambda_{k}$, попавших в сектор $T \cap B(0, r)$, с учетом их кратностей $m_{k}$. Согласно известному результату Б. Я. Левина (см. [22, гл. 3, §3, теорема 4]), правильная распределенность последовательности при порядке один равносильна тому, что она совпадает (с учетом кратности) с нулевым множеством целой функции экспоненциального типа и вполне регулярного роста.

Последовательность $\Lambda=\left\{\lambda_{k}, m_{k}\right\}_{k=1}^{\infty}$ будем называть правильной, если она является частью правильно распределенной последовательности при порядке один, т. е. если $\Lambda$ является частью нулевого множества (с учетом кратностей $m_{k}$ ) целой функции экспоненциального типа и вполне регулярного роста. Пусть $\Lambda-$ правильная последовательность. Через $F(\Lambda)$ обозначим множество всех целых функций экспоненциального типа и вполне регулярного роста, для каждой из которых $\Lambda$ является частью ее нулевого множества.

Следуя работам [4], [14], для последовательности $\Lambda=\left\{\lambda_{k}, m_{k}\right\}_{k=1}^{\infty}$ введем величину, характеризующую меру сгущения точек $\lambda_{j}$ вокруг $\lambda_{k}$. Положим $S_{\Lambda}=0$, если $\Lambda$ состоит из конечного числа элементов, и

$$
S_{\Lambda}=\lim _{\delta \rightarrow 0} \underset{k \rightarrow \infty}{\lim } \frac{\ln \left|q_{\Lambda}^{k}(\delta)\right|}{\left|\lambda_{k}\right|}
$$

в противном случае. Здесь

$$
q_{\Lambda}^{k}(\delta)=\prod_{\lambda_{j} \in B\left(\lambda_{k}, \delta\left|\lambda_{k}\right|\right), k \neq j}\left(\frac{\lambda_{j}-\lambda_{k}}{3 \delta\left|\lambda_{j}\right|}\right)^{m_{j}} .
$$

Величина $S_{\Lambda}$ схожа по смыслу с классическим индексом конденсации Бернштейна-Леонтьева последовательности $\Lambda$ (см. [2]) и играет ту же роль, что и последний, при исследовании особых точек суммы ряда (1) (см. [18]).

Пусть последовательность $\Lambda=\left\{\lambda_{k}, m_{k}\right\}_{k=1}^{\infty}$ такова, что система $E(\Lambda)$ неполна в пространстве $H(D)$. На пространстве $Q(D)$ определим оператор $L$ следующим образом. Последовательности $d=\left\{d_{k, n}\right\} \in Q(D)$ (возможно, не каждой) поставим в соответствие сумму $g_{d}(z)$ ряда $(1)$, сходящегося в топологии пространства $H(D)$. Таким образом, оператор $L$ действует из пространства $Q(D)$ в пространство $W(D, \Lambda)$. При этом область его определения не обязана совпадать с $Q(D)$. Пусть $W$ - замкнутое инвариантное относительно оператора дифференцирования подпространство в $H(D)$ со спектром $\Lambda$, допускающее 
спектральный синтез. Другими словами, подпространство $W$ совпадает с замыканием в $H(D)$ линейной оболочки системы $E(\Lambda)$. Следовательно, оно содержит подпространство $W(D, \Lambda)$. Таким образом, оператор $L$ действует из пространства $Q(D)$ в $W$. Неполнота системы $E(\Lambda)$ в пространстве $H(D)$ равносильна тому, что $W$ является нетривиальным подпространством в $H(D)$.

Теорема 3. Пусть $D$ - ограниченная выпуклая область в $\mathbb{C}$, a $W$ - нетривиальное замкнутое и инвариантное относительно оператора дифберенцирования подпространство в $H(D)$ со спектром $\Lambda$, допускающее спектральный синтез. Тогда следующие утверждения эквивалентны:

1. Оператор $L$ является изоморфизмом линейных топологических пространств $Q(D)$ и $W$.

2. Каждая функиия из $W$ представляется рядом (1), равномерно сходящимся на компактах из области $D$.

3. $S_{\Lambda}=0, \Lambda$ - правильная последовательность и существует функиия $f \in F(\Lambda)$, такая, что

$$
h_{f}(\lambda)=H_{D}(\lambda), \quad \lambda \in \mathbb{C} .
$$

Доказательство. $1 \Longrightarrow 2$ Если верно утверждение 1 , то оператор

$$
L: Q(D) \rightarrow W
$$

сюръективен. Согласно определению последнего, это означает, что каждая функция из $W$ представляется рядом (1), равномерно сходящимся на компактах из области $D$, т. е. выполнено 2.

$2 \Longrightarrow 1$. Пусть верно утверждение 2. Тогда, согласно определению подпространства $W(D, \Lambda)$, оно содержит $W$. Выше отмечалось, что в условиях теоремы имеет место и обратное вложение $W(D, \Lambda) \subset W$. Таким образом, подпространства $W(D, \Lambda)$ и $W$ совпадают. В частности, это означает, что $W(D, \Lambda)$ замкнуто в $H(D)$. Кроме того, $W$ нетривиально, а потому система $E(\Lambda)$ неполна в $H(D)$. Тогда по теореме 1 верно равенство $m(\Lambda)=0$. В начале предыдущего параграфа мы отмечали, что неполнота системы $E(\Lambda)$ влечет за собой также равенство $\sigma(\Lambda)=0$.

В силу утверждения 2 каждая функция $g \in W$ представляется в области $D$ рядом (1), сходящимся в топологии пространства $H(D)$. Тогда по теореме 2 последовательность $d=\left\{d_{k, n}\right\}$ коэффициентов этого представления является элементом пространства $Q(D)$ (и даже более узкого пространства $Q(D(\Theta(\Lambda)))$ ). Следовательно, оператор $L: Q(D) \rightarrow W$ сюръективен. Он также и инъективен. Действительно, в начале $\S 2$ было замечено, что неполнота системы $E(\Lambda)$ в пространстве $H(D)$ влечет за собой существование последовательности функционалов $\left\{\mu_{k, n}\right\} \subset H^{*}(D)$, биортогональной к системе функций $E(\Lambda)$. Поэтому коэффициенты разложения функции $g \in W$ в ряд (1), равномерно сходящийся на компактах из области $D$, определяются однозначно по формуле

$$
d_{k, n}=\left(\mu_{k, n}, g\right), \quad k=1,2, \ldots, n=0,1, \ldots, m_{k} .
$$

Отметим, что $W$ как замкнутое подпространство пространства Фреше $H(D)$ (см., например, [1]) само является пространством Фреше. Топология в $W$ задается при помощи системы полунорм

$$
\|g\|_{p}=\sup _{z \in K_{p}}|g(z)|, \quad p=1,2, \ldots,
$$


где $\left\{K_{p}\right\}_{p=1}^{\infty}=K(D)$. Используя теорему 2 для каждой функции $g \in W$ и каждого $p=1,2, \ldots$, получаем

$$
\|g\|_{p}=\sup _{z \in K_{p}}|g(z)| \leqslant \sum_{k=1, n=0}^{\infty, m_{k}-1}\left|d_{k, n}\right| \sup _{z \in K_{p}}\left|z^{n} \exp \left(z \lambda_{k}\right)\right| \leqslant C_{p}\|d\|_{p+2},
$$

где $\left\{d_{k, n}\right\}$ - последовательность коэффициентов разложения функции $g$ в ряд (1), а постоянная $C_{p}>0$ зависит от номера $p$, но не зависит от $d=\left\{d_{k, n}\right\}$, a значит, и от $g$.

Таким образом, оператор $L: Q(D) \rightarrow W$ является взаимно однозначным линейным и непрерывным отображением пространств Фреше. Тогда по теореме об открытом отображении (см. [20, приложение 1, теорема 2]) для пространств Фреше оператор $L$ осуществляет изоморфизм линейных топологических пространств $Q(D)$ и $W$. Это дает нам утверждение 1.

$2 \Longrightarrow 3$. Если верно утверждение 2 , то, как и выше, $m(\Lambda)=0$. Тогда по теореме 5.2 из [4] верно также следующее утверждение: $S_{\Lambda}=0$ и существует целая функция экспоненциального типа $f$, которая обращается в нуль в каждой точке $\lambda_{k}$ с кратностью, не меньшей чем $m_{k}$, имеет регулярный рост и ее верхний индикатор равен $H_{D}$, т. е. имеют место тождества $\underline{h}_{f} \equiv h_{f} \equiv H_{D}$. Первое тождество здесь означает, что функция $f$ имеет регулярный рост. Таким образом, $\Lambda$ - правильная последовательность, $F \in f(\Lambda)$ и

$$
h_{f}(\lambda)=H_{D}(\lambda), \quad \lambda \in \mathbb{C} .
$$

Тем самым мы показали, что утверждение 3 верно.

$3 \Longrightarrow 2$. Пусть верно утверждение 3 . Тогда $S_{\Lambda}=0$ и, согласно определению правильной последовательности, существует целая функция экспоненциального типа $f$, которая обращается в нуль в каждой точке $\lambda_{k}$ с кратностью, не меньшей чем $m_{k}$, имеет регулярный рост (т. е. $\left.\underline{h}_{f} \equiv h_{f}\right)$ и выполнено равенство

$$
h_{f}(\lambda)=H_{D}(\lambda), \quad \lambda \in \mathbb{C} .
$$

Тогда по теореме 5.2 из [4] любая функция $g \in W$ представляется рядом (1), который сходится в каждой точке области $D$. Согласно же теореме 2 этот ряд будет сходиться также в топологии пространства $H(D)$. Это дает нам утверждение 2. Теорема доказана.

\section{ЛитЕРАТУРА}

[1] В. В. Напалков, Уравнения свертки в многомерных пространствах, Наука, М., 1982.

[2] А. Ф. Леонтьев, Рядъ экспонент, Наука, М., 1976.

[3] И. Ф. Красичков-Терновский, Однородное уравнение типа свертки на выпукльх областях, ДАН СССР, 197:1 (1971), 29-31.

[4] А. С. Кривошеев, Фундаментальный принцип для инвариантнъх подпространств в выпуклых областях, Изв. РАН. Сер. матем., 68:2 (2004), 71-136.

[5] G. Valiron, Sur les solutions des équations différentielles linéaires d'ordre infini et à coefficients constants, Ann. Sci. Ecole Norm. Sup., 46:1 (1929), 25-53.

[6] L. Schwartz, Théorie générale des fonctions moyenne-périodique, Ann. Math., 48:4 (1947), 857-929. 
[7] А. О. Гельфонд, Линейные дифференциалъные уравнения с постоянными коэффициентами бесконечного порядка и асимптотические периоды целых функиий, Труды МИАН СССР, т. 38, Изд-во АН СССР, М., 1951, 42-67.

[8] D. G. Dickson, Expansions in series of solutions of linear difference-differential and infinite order differential equations with constant coefficients, Mem. Amer. Math. Soc., 1957:23 (1957), 1-72.

[9] Б. Я. Левин, О некоторых приложениях интерполяиионного ряда Лагранжа $к$ теории целых функиий, Матем. сб., 8:3 (1940), 437-454.

[10] Ю. Ф. Коробейник, Интерполяиионные задачи, нетривиальные разложения нуля u представляющие системы, Изв. АН СССР. Сер. матем., 44:5 (1980), 1066-1114.

[11] Ю. Ф. Коробейник, Представляющие системы, УМН, 36:1 (1981), 73-126.

$[12]$ А. В. Братищев, Ю. Ф. Коробейник, Интерполяиионнал задача в пространствах целых функиий заданного уточненного порлдка, Изв. АН СССР. Сер. матем., 40:5 (1976), 1102-1127.

[13] А. В. Братищев, Базисы Кете, целье функиии и их приложения, Дисс. д.ф.-м.н., Ростов-на-Дону, 1995.

[14] А. С. Кривошеев, Критерий фундаменталвного принципа для инвариантных подпространств, Докл. РАН, 389:4 (2003), 457-460.

[15] А. Ф. Леонтьев, Целье функиии. Рлды экспонент, Наука, М., 1983.

[16] К. Лейхтвейс, Выпуклые множества, Наука, М., 1985.

[17] О. А. Кривошеева, Ряды экспоненииальных мономов в комплексных областлх, Вестник УГАТУ. Матем., 9:3(21) (2007), 96-103.

[18] О. А. Кривошеева, Особые точки суммъ рлда экспонент на граниче области сходимости, Уфимский матем. ж., 1:4 (2009), 78-109.

[19] В. В. Напалков, О. А. Кривошеева, Теоремы Абеля и Коши-Адамара для рлдов экспоненииальных мономов, Докл. РАН, 432:5 (2010), 594-596.

[20] А. П. Робертсон, В. Дж. Робертсон, Топологические векторные пространства, Мир, М., 1967.

[21] П. Лелон, Л. Груман, Целье функиии многих комплексных переменных, Мир, М., 1989.

[22] Б. Я. Левин, Распределение корней целых функиий, Гостехиздат, М., 1956.

Башкирский государственный университет

e-mail: kriolesya2006@yandex.ru

Поступило в редакцию

Институт математики с ВЦ УНЦ РАН

e-mail: shaig@anrb.ru 